\title{
A comparison of spectroscopic techniques for human breath analysis
}

\author{
Kam Kong Chow ${ }^{\mathrm{a}, \mathrm{b}}$, Michael Short ${ }^{\mathrm{a}}$ and Haishan Zeng $\mathrm{a,b,*}$ \\ ${ }^{a}$ Imaging Unit - Integrative Oncology Department, British Columbia Cancer Agency Research Centre, \\ Vancouver, BC, Canada \\ ${ }^{\mathrm{b}}$ Department of Physics and Astronomy, University of British Columbia, Vancouver, BC, Canada
}

\begin{abstract}
The analysis of human breath has been driven to new heights and has great potential to impact our society in the area of medical science. Breath analysis is promising as non-invasive, simple and point-of-care clinical measurements to reduce the medical burden caused by invasive, time-consuming and expensive clinical devices. Spectroscopic techniques for breath analysis can offer information to correlate its signals to exhaled substances for molecular identification and quantification to provide the pathophysiological status of the body. In this review paper, techniques such as mass spectrometry-based (gas chromatography-mass spectrometry, proton transfer reaction-mass spectrometry, selected ion flow tube-mass spectrometry), laser absorption spectroscopy-based (cavity ring down spectroscopy and tunable diode laser absorption spectroscopy) and other spectroscopic techniques for breath analysis applications are compared in terms of its advantages/disadvantages, versatilities and plausibility to be transformed in clinical applications.
\end{abstract}

Keywords: Breath analysis, volatile organic compounds (VOCs), exhaled substances, spectroscopy, mass spectrometry, laser spectroscopy

\section{Introduction}

In the ancient days, Hippocrates instructed his students to smell patients' breaths. For example, fruity smell breath can mean high risk of diabetes and fishy smell breath may point the finger to liver impairment [45]. Exhaled breath contains mainly nitrogen, oxygen, small amount of carbon dioxide and other substances in trace amounts [51]. These substances, which constitute a distinctive "breathprint", are in trace amounts and occur because of various biochemical processes in human body. By correctly analyzing the exhaled "breathprint", the physiological and pathophysiological states of the body can be revealed and provide results quicker than conventional body fluid tests [38]. Blood and urine tests both have been serving the medical industry for years. Scientists and physicians have been relying on these body fluid tests to check the status of human body such as disease diagnosis, drug test, etc. However, these reliable techniques require sample collection, which some consider as invasive and inconvenient, and is not suitable for on-line measurements and real-time monitoring. Off-line sampling is considered as relatively time-consuming since it requires extra work for analysis. Breath analysis has the potential to benefit the medical service as it can provide point-of-care, cost-effective and painless means to work in routine clinical tests and monitoring.

\footnotetext{
*Corresponding author: Haishan Zeng, Imaging Unit - Integrative Oncology Department, British Columbia Cancer Agency Research Centre, 675 West 10th Avenue, Vancouver, BC, Canada V5Z 1L3. Tel.: +1 604675 8083; Fax: +1 6046758099 ; E-mail: hzeng@bccrc.ca.
} 
Table 1

Common diseases with example correlated exhaled biomarkers

\begin{tabular}{llc}
\hline Disease & \multicolumn{1}{c}{ Related exhaled biomarker } & Reference \\
\hline Asthma & Nitric oxide & {$[2,39,40]$} \\
H. pylori infection & ${ }^{13} \mathrm{C} /{ }^{12} \mathrm{C}$ of carbon dioxide & {$[9,54]$} \\
Lipid peroxidation & Ethane & {$[10]$} \\
Lung cancer & Benzene, ethane, formaldehyde, isoprene, propanol, etc. & {$[1,34,58,63,76]$} \\
Renal disease & Ammonia, isoprene & {$[11,12,29,41]$} \\
Diabetes & Acetone & {$[13,73]$} \\
\hline
\end{tabular}

The first detailed quantitative study of human breath analysis was done in 1970s using gas chromatography (GC) by Pauling et al. [44]. After Pauling's finding, Phillips et al. in their study stated that there are over three thousand different substances in human exhaled breath analysis [48]. Various types of major spectroscopic techniques have been used to study the analysis of human breath, including mass spectrometry (MS) related technologies [12,27,47] and laser absorption spectroscopy (LAS) $[40,41,70]$, etc. It turns out that these exhaled substances are mostly volatile organic compounds (VOCs) like alcohols, ketones, ester and some small volatile inorganic compounds such as $\mathrm{NO}, \mathrm{NH}_{4}$. Some volatile compounds are known to appear in blood and to be able to cross the alveolar membrane in the lung and thus present in expired breath. Studies have shown that the concentrations of certain exhaled endogenous volatile compounds can be important indicators of the pathophysiological states and the exhaled biomarkers of certain diseases to distinguish healthy and diseased state $[23,31,36,47,56]$. Table 1 shows examples of potential exhaled biomarkers of common diseases.

VOCs are always present in our planet, released from both natural and artificial emission. Our body absorbs and generates lots of VOCs by various means at all times. Most VOCs can be found in exhaled breath in the range of parts per trillion, parts per billion to parts per million by volume (pptv, ppbv to ppmv). It has been shown that there are many types of VOCs present in breath and the concentration of a particular VOC could be different from person to person [48]. Some biomarkers may even show different concentrations in breath due to age or/and gender, e.g., isoprene [25,61]. It is important to note that the exhaled biomarker and the corresponding disease are not necessary one to one. Meaning that a disease can have multiple exhaled biomarkers, and a chemical substance can be biomarkers of multiple diseases.

\section{Breath sample preparation}

Not all analytical tools support on-line breath analysis, and sample preparation may be needed for some analytical tools, for example, pre-concentration of breath sample and liquid phase breath condensate.

Solid-phase microextraction (SPME) was developed in 1990s [16] and it is one of the preconcentration tools used most frequently in VOCs analysis combined with gas chromatography-mass spectrometry (GC-MS). SPME contains fibre that is adhesive to certain types of VOCs and the fibre can in principle absorb targeted molecules based on the proportion once the equilibrium is reached. Heating is then used to desorb the VOCs from the fibre. The fact that it is convenient and inexpensive allows researchers to selectively enhance the concentration of particular types of VOC. There are SPME injection ports available to integrate with gas chromatography (GC) to simplify the thermal desorption procedures. SPME with mass spectrometry techniques used in breath analysis has been employed in various 
studies $[16,20,77]$. Sensitivity for certain VOCs in human breath can be further improved by integrating low-temperature glassy carbon with SPME [15].

Exhaled breath condensate (EBC) is the collection of breath in liquid phase, such that water vapor and some gas phase exhaled substance in the breath sample can be turned into liquid phase via a cooled trap. However, not all substances in EBC have concentrations correlated to those in direct breath measurements [5]. The solubility of the exhaled substances can directly influence the workability of EBC. Therefore, the concentration of substances sampled by EBC with low solubility in water may experience difficulties to be accurately correlated to those in direct breath measurements. EBC can be analyzed with, for example, a headspace integrated MS-based analytical tool for off-line analysis.

Most of the off-line breath analyses are done with gas phase breath and balloon-like sampling bags are often used for collection. Off-line sampling plus pre-concentration is possible to increase the sensitivity of the instrument but it may also lead to potential error due to sample contamination. Calibrations and cleaning are required to make sure endogenous VOCs from human body can be distinguished. On-line sampling, which breath sample is collected and analyzed at the same place, would generally be more accurate and time-efficient compared to off-line sampling.

\section{Techniques for breath analysis}

Various forms of breath analyzing techniques have been designed for different purposes: An alcohol breath analyzer is commonly used worldwide to deter the operation of a motor vehicle by an impaired driver. It is purposely designed for the blood alcohol level testing only and has no way to work for other types of exhaled substances. Electronic nose [30], is an interesting and cost-effective development. The semiconductor arrays can generate series of electronic signatures from the analyte but it has difficulty to give accurate quantifiable information to correlate the measured analytes. Interestingly enough, researchers have demonstrated breath analysis for lung cancer patients by trained dogs [35], but this approach may not be easily translated into clinical settings. These non-spectroscopic methods for breath analysis do not give correlated signals to specify the analytes and therefore cannot be used for molecular identification purpose.

Spectroscopic breath analysis tools offer advantages for the identification and quantification of the exhaled substances. There are two major types of spectroscopic methods for breath analysis, mass spectrometry and laser spectroscopy. Mass spectrometry has been widely accepted as a powerful analytical technique that measures the mass-to-charge $(\mathrm{m} / z)$ ratio of charged molecules, and it has a historical term called mass spectroscopy. Laser spectroscopy is a general term to describe analytical measurements based on optical-medium interactions such as photon absorption and scattering using a laser source. In this paper, different spectroscopic techniques are reviewed with its advantages, limitations and potential in breath analysis applications.

\subsection{Various forms of mass spectrometry}

Mass spectrometry (MS) has long been playing an important role in various fields, such as food, medicine, forensic, sports, etc, for the identification and quantification of unknown substances. For the trace substances in expired breath to be detectable and truly quantified, other technologies have been combined with MS, such as gas chromatography-MS (GC-MS) [46,47], proton transfer reaction-MS (PTR-MS) [8,50], selected ion flow tube-MS (SIFT-MS) [12,62]. Table 2 shows examples of breath analysis done by MS-based techniques. 
Table 2

Examples of MS-based analytical methods for breath analysis

\begin{tabular}{lllcc}
\hline MS type & \multicolumn{1}{c}{ Medical condition } & \multicolumn{1}{c}{ Studied molecules } & Level of concentration measured & Reference \\
\hline GC-MS-SPME & Lung cancer & 21 VOCs & $>10^{-1} \mathrm{ppbv}$ & {$[1]$} \\
& Lung cancer & $22 \mathrm{VOCs}$ & $\mathrm{N} . \mathrm{A}$ & {$[47]$} \\
& Diabetes & Acetone & $>10^{2} \mathrm{ppbv}$ & {$[13]$} \\
PTR-MS & Lung Cancer & Formaldehyde, propanol & $>10^{0} \mathrm{ppbv}$ & {$[76]$} \\
& Renal disease & Isoprene & $>10^{1} \mathrm{ppbv}$ & {$[29]$} \\
& Lipid-lowering therapy & Isoprene & $>10^{-1} \mathrm{ppbv}$ & {$[50]$} \\
& Anaesthetic monitoring & Propofol & $>10^{0} \mathrm{ppbv}$ & {$[8]$} \\
SIFT-MS & Renal disease & Isoprene & $>10^{1} \mathrm{ppbv}$ & {$[12]$} \\
& Renal disease & Ammonia & $>10^{2} \mathrm{ppbv}$ & {$[11]$} \\
& Fasting & Acetone, ammonia, ethanol, & $>10^{1} \mathrm{ppbv}$ & {$[60]$} \\
& & isoprene, methanol & & \\
\hline
\end{tabular}

\subsubsection{Gas chromatography-mass spectrometry $(G C-M S)$}

GC-MS is one of the most useful analytical techniques in breath analysis $[1,13,46,47]$. The name "gold standard" was assigned to GC-MS in scientific analysis because of its outstanding analytical power and widely used in various areas to offer reliable substance identification and quantification.

Gas chromatography (GC) is one of the most common pre-separation techniques combined with MS and it separates molecules into different retention times. The advantage of the temporal separation of molecules leads GC-MS capable to analyze complex gas mixtures. However, it reduces the possibility of real-time measurements due to the slow turnaround time because GC may take up to tens of minutes for gas mixture to be separated in the capillary column. Moreover, water vapor in breath is a problem as it can interfere with the chromatogram and damage the GC capillary column [6].

GC-MS gives detailed information of the analyte but it generally requires sample pre-concentration to increase the sensitivity of the device, such as SPME. GC-MS-SPME can usually accurately identify compounds with certainty. However, contamination of the sample during the manipulation could be a problem. This pre-concentration method may involve competitive adsorption on the SPME-fibre [7], which may result GC-MS-SPME being only semi-quantificational and thus introduces the need of an extra calibration or analytical device for a true molecular quantification. Moreover, even with the help of SPME, GC-MS is still relatively insensitive compared to other MS-based techniques $[1,26]$. The preconcentration method also reduces the time resolution, so it is rather a slow method in breath analysis among other MS-related breath analyzers.

On the other hand, the traditional electron impact ionization has led to difficulties in the identification of complex molecules due to the extensive fragmentations. Difficult to resolve the presence of isobaric compounds and structural isomers are the other intrinsic shortcomings. Due to its working mechanism, molecules in human breath with the same $\mathrm{m} / \mathrm{z}$ ratios are difficult to be distinguished, for example ketones and aldehydes [26].

Figure 1 shows an example of total ion chromatograms of human exhaled breath samples (a control healthy subject and a lung cancer patient). The chosen potential biomarkers (1-butanol and 3-hydroxy2-butanone) were checked by their mass spectrums and retention times with those of the standards. The analysis was done in the following MS conditions: full-scan mode, $\mathrm{m} / \mathrm{z}$ ratio 35-350 and $15 \mathrm{~min}$ for collection time [63]. The pink curve (lower curve) is from the healthy sample, and the black curve (upper curve) is from the lung cancer patient. Figure 1(b) is zoomed to show the spectral range for the 

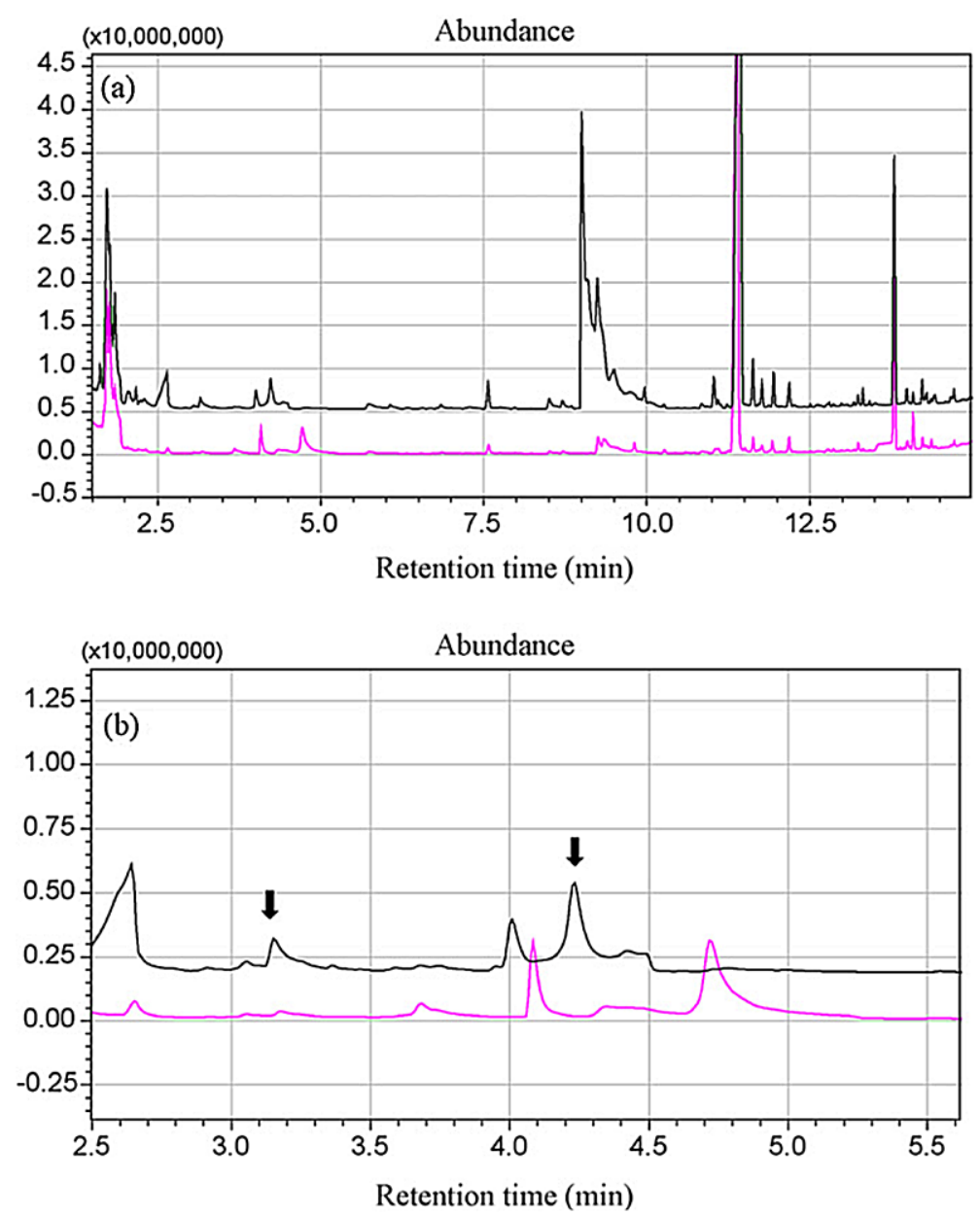

Fig. 1. (a) Example of total ion chromatograms of exhaled breath samples taken from a healthy control (pink, lower curve) and a lung cancer patient (black, upper curve), analyzed with GC-MS to find the composition of the breath. (b) The amplified chromatogram showing 1-butanol (retention time $3.15 \mathrm{~min}$ ) and 3-hydroxy-2-butanone (retention time $4.18 \mathrm{~min}$ ) with noticeable higher peak height in lung cancer patient's breath sample, pointed by arrows. (Reprinted from reference [63] with permission from Elsevier). (The colors are visible in the online version of the article; http://dx.doi.org/10.3233/BSI-120029.)

two selected biomarkers. The signals for both the 1-butanol (retention time $3.15 \mathrm{~min}$ ) and 3-hydroxy2-butanone (retention time $4.18 \mathrm{~min}$ ) from the lung cancer patient are higher than that of the healthy control.

\subsubsection{Proton transfer reaction-mass spectrometry (PTR-MS)}

PTR-MS for on-line measurements of VOCs was introduced in the 1990s and has been increasing rapidly in usage since then $[4,17,27,28]$. The sensitivity of PTR-MS was in the ppbv level [17], but researchers had brought the limit down to pptv level [27].

PTR-MS differs from the traditional MS mainly by the way of sample ionization, in which hydronium ion $\left(\mathrm{H}_{3} \mathrm{O}^{+}\right)$is used for the ionization instead of the electron impact. The sample gaseous molecules receive a proton from the donor $\left(\mathrm{H}_{3} \mathrm{O}^{+}\right)$, and thereby becoming ionized. This "soft" proton ionization significantly reduces the fragmentation compared to the conventional energetic electron impact, which is one of the major advantages of PTR-MS over GC-MS. However, this leads to an intrinsic limitation 
of PTR-MS in which it can only work for target molecules with a proton affinity (PA) higher than that of water. Detailed study and a list of the proton affinities of common VOCs can be found from Ref. [19].

On the other hand, PTR-MS can provide explainable spectra of complex gas mixtures such as breath sample without pre-concentration or pre-separation procedures. However, PTR-MS may still experience signal overlapping issues, studies have suggested that the proportion of the fragments produced from compounds with the same $\mathrm{m} / \mathrm{z}$ ratio should be taken into account to obtain correct quantification [55]. Lacking of certainty in gas molecule identification is another shortcoming of PTR-MS, researchers have suggested that PTR-MS studies may need a larger group size compared to that of GC-MS to compensate the molecular identification uncertainty [1].

The PTR-MS technique generally has a response time less than $1 \mathrm{~s}$, the total analysis time could be in minutes which allows on-line and real-time measurements [21,37]. Commercial product is available by Ionicon Analytik GmbH with real-time, $<1$ pptv limit of detection (LOD) and detectable mass up to $500 \mathrm{amu}$. It also offers switchable precursor ions to minimize the inherent limitation of the sample's PA.

\subsubsection{Selected ion flow tube-mass spectrometry (SIFT-MS)}

The application of SIFT-MS on trace gas analysis in breath has been studied in 1990s [65-67]. Studies of SIFT-MS have shown potential breath analysis applications in disease diagnosis, pharmaceutical monitoring and physiological studies [59,68,69].

SIFT-MS shares similar advantages of PTR-MS, but it makes use of more choices for the precursor ions for chemical ionization: $\mathrm{H}_{3} \mathrm{O}^{+}, \mathrm{NO}^{+}$, or $\mathrm{O}_{2}^{+}$. The fact that SIFT-MS can supply multiple precursor ions offers the ability to work with a wider range of exhaled VOCs and molecules with the same $\mathrm{m} / \mathrm{z}$ ratio. It is because of the different chemical reactions offered by different precursor ions to the target ions. For example, acetone and propanol are isobaric VOCs commonly found in human exhaled breath. These two VOCs have different ion chemistries to $\mathrm{H}_{3} \mathrm{O}^{+}$and $\mathrm{NO}^{+}$as a result producing distinctive adduct ions separable in SIFT-MS [65]. However, there are some compounds that SIFT-MS cannot efficiently detect due to their high ionization energies and low proton affinities, such as hydrogen gas and simple hydrocarbons with low molecular mass [59].

Furthermore, SIFT-MS is able to perform self-calibration based on the absolute humidity of the expired breath. This is due to the internal formation of the hydrates of hydronium ion. By comparing the signal levels of $\mathrm{H}_{3} \mathrm{O}^{+}$to its hydrates, water vapour content in the sample can be analyzed and thus allows internal standardization for breath analysis [69].

SIFT-MS generally can detect trace substances in pptv level, it allows absolute quantification [12] and there is no sample pre-concentration requirement for modern SIFT-MS to achieve high sensitivity. Real-time analysis and on-line mode are possible to achieve in breath to breath resolution. Commercial product for breath analysis is available by Syft Technologies Ltd with the LOD and response time in tens of pptv and tenth of seconds respectively.

\subsubsection{Comparison of MS-based techniques}

GC-MS for the analysis of gaseous sample is still widely used and reliable. The pre-separation procedure allows GC-MS to work for complex gas mixtures, but at the same time prevents it from real-time and on-line breath sampling. Pre-concentration method such as SPME is often used to increase the sensitivity but may lead to potential contamination and semi-quantification. The traditional electron ionized fragmentations would complicate the spectral analysis of isobaric VOCs.

PTR-MS can overcome some of the technical problems that GC-MS has. PTR-MS does not need sample pre-concentration nor pre-separation procedure, it is therefore possible for on-line and real-time breath measurements. But PTR-MS has smaller mass detection range compared to GC-MS. PTR-MS 
generally has higher sensitivity than SIFT-MS and GC-MS but it has maximum detectable concentration only up to ppmv level. The small amount of fragmentations of analyte makes it easier for the analysis of complex molecules. However, the use of hydronium ion as the only precursor may hinder its ability to distinguish molecules with the same $\mathrm{m} / \mathrm{z}$ ratio and failed to ionized molecules with PA lower than that of water. PTR-MS does not easily identify molecule with certainty, which is one of the disadvantages of this technique [1].

SIFT-MS, with more choice in precursor ions than PTR-MS, shares similar advantages of PTR-MS such as: low fragmentation, pre-concentrated sample unnecessary for on-line and real-time measurements. It has been the alternative tool for the analysis of isobaric ions, where PTR-MS and GC-MS are not a perfect candidate. However, the trade off for this increase in detection variety is resulting lower LOD of SIFT-MS. Recent study has shown the feasibility for PTR-MS to work with more choices of precursor ions without reducing its high sensitivity [21]. But the rapid-switching (milliseconds) multiple precursor ions offered by SIFT-MS would be still superior to the modified PRT-MS system because it takes time to switch the ion source gas mixture [69]. Another advantage of SIFT-MS over other MS techniques is its ability to self-calibrate by breath humidity.

Most MS-based breath analyses are undertaken in research laboratory settings. Although there are MS field units, they are specifically designed for certain types of target molecules for only limited use. Researchers and commercial companies have been attempting to reduce the size and cost of MS-based analytical tools. However, these are still intrinsic problems with MS which hinder its mobility and applications into frontline clinical settings.

\subsection{Laser absorption spectroscopy (LAS)}

LAS techniques, which measure the photon absorption properties of an analyte, have been known for decades but have only become available for trace amount detection for breath analysis recently. Due to the fast development of semiconductor lasers and photodetectors, LAS has undergone recent advancements that enable sufficient sensitivity and selectivity for real-time breath analysis [32,57,71], such as cavity ring down spectroscopy (CRDS) [33,43], tunable diode laser absorption spectroscopy (TDLAS) [39,52] and photoacoustic spectroscopy (PAS) [41]. Table 3 shows examples of breath analysis done by LAS-based techniques.

\subsubsection{Cavity ring down spectroscopy (CRDS)}

The initial idea of CRDS was an experiment of light absorption in a multipass optical cavity reported in 1960s. Since then, CRDS has been widely investigated in environmental science, chemical composition analysis, and increasingly in breath analysis.

CRDS is a measurement of the absorption characteristics of gas samples with a high finesse optical resonator consisting of highly reflective and carefully aligned mirrors to enhance the effective optical interaction length. Photons bounce back and forth within the ultra-low-loss cavity and thus the interaction length can be increased more than 10,000-fold and hence offers significant higher number of photon-molecular interactions to enhance molecular absorptions [71]. This makes CRDS generally more sensitive than other multipass configurations and reaches pptv sensitivity level for breath analysis [43].

The ring-down decay time of CRDS is an indirect measurement of the photon leakage in the cavity in a particular absorption frequency to measure the concentration of a particular analyte. So the experiment can run for a long integration time to achieve high sensitivity with reproducible results independent to the laser fluctuation. 
Table 3

Examples of LAS-based analytical methods for breath analysis

\begin{tabular}{|c|c|c|c|c|c|c|c|}
\hline LAS-type & Laser type & $\begin{array}{c}\text { Absorption } \\
\text { peak(s) }\end{array}$ & $\begin{array}{c}\text { Cavity/effective } \\
\text { length }\end{array}$ & $\begin{array}{c}\text { Medical } \\
\text { condition }\end{array}$ & $\begin{array}{c}\text { Studied } \\
\text { molecules }\end{array}$ & $\begin{array}{c}\text { Level of conc. } \\
\text { measured }\end{array}$ & Reference \\
\hline CRDS & $\begin{array}{l}\text { Q-switch } \\
\text { Nd:YAG laser }\end{array}$ & $266 \mathrm{~nm}$ & $43 \mathrm{~cm} / \mathrm{N} . \mathrm{A}$ & Diabetes & Acetone & $>10^{3} \mathrm{ppbv}$ & [73] \\
\hline CLOAS & $\begin{array}{l}\text { CO-overtone } \\
\text { sideband laser }\end{array}$ & $3 \mu \mathrm{m}$ & $53 \mathrm{~cm} />3 \mathrm{~km}$ & Peroxidation & Ethane & $>10^{0} \mathrm{ppbv}$ & {$[10]$} \\
\hline $\begin{array}{l}\text { Off axis } \\
\text {-ICAS }\end{array}$ & $\begin{array}{l}\text { Quantum } \\
\text { cascade laser }\end{array}$ & $5.45 \mu \mathrm{m}$ & $50 \mathrm{~cm} / 700 \mathrm{~m}$ & Asthma & Nitric oxide & $>10^{1} \mathrm{ppbv}$ & {$[2]$} \\
\hline OFC-CEAS & $\begin{array}{l}\text { Mode-locked } \\
\mathrm{Er}^{+3} \text { laser }\end{array}$ & $1.5-1.7 \mu \mathrm{m}$ & N.A & N.A & $\begin{array}{l}\text { Carbon oxides, ammonia, } \\
\text { methane, water }\end{array}$ & $>10^{0} \mathrm{ppmv}$ & [70] \\
\hline TDLAS & $\begin{array}{l}\mathrm{Pb} \text {-salt } \\
\text { semiconductor }\end{array}$ & $5.2 \mu \mathrm{m}$ & $55 \mathrm{~cm} / 36 \mathrm{~m}$ & Asthma & Nitric oxide & $>10^{1} \mathrm{ppbv}$ & {$[39,40]$} \\
\hline TDLAS & $\begin{array}{l}\mathrm{Pb} \text {-salt tunable } \\
\text { diode laser }\end{array}$ & $3.4 \mu \mathrm{m}$ & N.A/170 m & Lung cancer & Ethane & $>10^{0} \mathrm{ppbv}$ & {$[58]$} \\
\hline PAS & $\mathrm{CO}_{2}$ laser & $\begin{array}{l}\text { 9-10 } \mu \mathrm{m} \\
\text { (with an } \\
\text { intracavity } \\
\text { grating) }\end{array}$ & N.A & Renal disease & Ammonia & $>10^{2} \mathrm{pptv}$ & [41] \\
\hline
\end{tabular}

With the advent of laser diode technology over the last decade, size of CRDS system is more compact and able to work in UV to mid-IR range for exhaled VOCs analysis. A portable device based on CRDS for breath acetone has been reported [72], and it is on-line and off-line feasible [73]. The UV and NIR region absorption peaks of acetone for diabetes monitoring [74]; and the advantages of the NIR wavelength region CRDS for the ${ }^{13} \mathrm{C} /{ }^{12} \mathrm{C}$ ratio test of $H$. pylori bacteria over the conventional isotope ratio mass spectrometer has been pointed out [9].

However, CRDS is generally untunable. The high reflectivity response only works in a narrow frequency range and it needs careful alignment and the spectral resolution is limited by the linewidth of the excitation laser. This limits the versatility of CRDS, as adjusting the cavity to work in other frequencies is cumbersome. The highly reflective mirrors $(99.99 \%)$ with a constant reflectivity across the spectral range are necessary to produce a reliable relationship between decay time (concentration) and wavelength (bonding information). In terms of the cavity design, the cavity length or the round trip time is limited by the lifetime of the excitation state such that the excited molecules are relaxed and ready for excitation again.

Variants of CRDS have been introduced to further improve the performance, for example: optical frequency comb technique is able to increase the spectral range for multiple molecules CRDS analysis. But yet, the multiplicity of CRDS is still incomparable to that of MS-based techniques.

\subsubsection{Tunable diode laser absorption spectroscopy (TDLAS)}

TDLAS is a combination of the traditional laser absorption techniques with tunable diode lasers, which measures the absorption characteristics of the sample molecules by scanning across its particular central absorption peak.

TDLAS can give not only the sample concentration information but also the temperature and pressure of the gas analyte. TDLAS can be integrated with a Herriott cell (multipass) consisting of concave mirrors. Unlike the high finesse optical cavity of CRDS, the laser beam comes out of the Herriot cell unparallel to the incoming beam. As its effective beam path is considerably shorter than CRDS, Her- 
riott cell can lengthen the effective absorption path by 10 - to 100 -fold and a LOD reaches the order of $10^{1}$ pptv [71].

Routine calibration using certified standard gas is usually required, but recent advancements have achieved self-calibration using the exhaled carbon dioxide $\left(\mathrm{eCO}_{2}\right)$ [39,52]. Commercial products based on TDLAS breath analysis for molecular detection in the mid-IR range are available by Ekips Technology Inc. For example, one of their breathmeters can detect NO in real-time measurement with the LOD in ppbv level.

\subsubsection{Photoacoustic spectroscopy (PAS)}

Photoacoustic spectroscopy (PAS) is a spectroscopic technology based on the indirect measurement of photon-molecule resonance (which converts light energy into sound energy). The setup usually involves microphones to detect the sound waves produced during photon absorption by molecules. The laser beam is not directly measured and, therefore, the background noise is relatively weaker compared to the conventional laser-based spectroscopy. This reduces the complications from finding the week absorption signals from a large background noise. Researchers have been applying PAS into human breath analyses for different exhaled biomarkers such as ammonia for renal disease [41]. Commercial product for research purpose is available from Pranalytica Inc., a breath analyzer for ammonia at ppb level.

\subsubsection{Waveguide assisted-LAS}

Capillary fibre is a hollow waveguide that provides light guidance with a hollow core fillable with analyte. Reflective material can be coated in the inner surface to provide better light transmission. Hollow waveguide assisted LAS for methane gas analysis has been investigated by researchers and the LOD they have achieved is few hundreds of ppbv [22].

\subsubsection{Comparison of LAS-based techniques}

CRDS consists of an ultra-low-loss cavity and, due to the long effective path length, generally offers higher sensitivity (pptv) than other LAS-based techniques. It indirectly measures the decay rate of an absorption frequency and thus overcomes the laser fluctuation problem. However, the versatility is limited by the high finesse cavity. CRDS is hard to multiplex and simultaneously measure multiple absorption peaks (samples). It is untunable and can only work in a narrow frequency range. The adjustment of the high finesse cavity is inconvenient, and the requirement of a tunable laser is not cost-effective. With the advent of laser diode technology, the size of CRDS system is more compact and able to work for UV to mid-IR range.

TDLAS provides alternative LAS solution of breath analysis and is very selective as the laser frequency can tune across the target peaks. The working mechanism offers temperature and pressure measurements, which are the unique features for TDLAS. Multipass configuration such as a Herriott cell can be integrated to increase the sensitivity to $10^{1} \mathrm{pptv}$ and most breath analysis applications are done in the mid-IR range.

PAS provides indirect measurement of the photon absorption characteristic via the photoacoustic effect. This technique offers the advantage of omitting the large laser background but rather less sensitive compared to CRDS due to the low photoacoutics conversion efficiency. Studies showed that the LOD can be improved by introducing a quartz tuning fork to suppress background noise [24].

Hollow waveguide to replace conventional gas cell design is a new idea. It offers the advantage for photons and the analyte to be confined in a small area to the increase number of interactions. However, the LOD in a single pass configuration is still not sensitive enough for sub-ppbv endogenous VOCs analysis. 
Like the MS-based methods, each LAS-based technique has its own advantages and limitations, and they complement each other. Failure or difficulty with multiplexing is one of the biggest challenges for LAS-based breath analysis since one disease/disorder can contain multiple biomarkers that have well separated absorption lines. With the advent of laser diodes and photoelectronics, different new techniques integrated LAS-based breath analyzers with better functionality and lower cost may have an important role in the future.

\subsection{Other spectroscopic techniques}

\subsubsection{Waveguide-assisted Raman scattering}

Raman scattering is an inelastic photon-medium scattering based on the unique molecular vibrations of the analyte. Molecule can be excited by a photon but relaxed into different vibrational energy states causing unique frequency-shifted photon emissions (fingerprint-type) that can be used for molecular identification. Hollow capillary fibre with internal reflective coating can be treated as a waveguide and at the same time as a gas cell based on Raman scattering [42]. However, these are still in the development stage and need much improvement in the transmission losses to lower the LOD for trace amount necessary for VOCs measurements.

\subsubsection{Ion mobility spectrometry}

Ion mobility spectrometry (IMS) refers to the measurements of the gas phase mobility of the analytes. Ion mixtures are driven by electric field but experience a high number of collisions with carrier gas along the drift way. The drifting time of particular species is dependent of its mass and geometric shape. Ion mixtures can therefore be separated according to its mobility and thus time-dependent signals can be obtained. IMS emphasizes low technical expenditure, fast response time and greater potential downsizing to a handheld device. However, the resolution of IMS is usually not satisfying, pre-separation step can be integrated such as multi-capillary column (MCC) $[3,53]$.

\subsection{Summary of spectroscopic methods}

Most of the early explorations of human breath analysis were done with MS-based techniques in the last few decades. The analytical power and sensitivity are usually the advantages of MS-based technologies. However, cost, size and weight always hinder its application to expand extensively. Downsizing and cost reduction are possible if the machine is designed only to focus on few selected disease biomarkers for limited clinical application. LAS-based tools for breath analysis are usually much cheaper and simpler than MS-based type. Most LAS analytical tools are real-time feasible as its multipass configuration and the application of light allows it to collect large amount of data instantly. However, a major shortcoming of LAS is that it can only work for a narrow spectral window and therefore it limits the detection to individual compound/peak only. Furthermore, expensive tunable laser and time consuming cavity re-alignment are usually necessary for different absorption regimes, which limits the plausibility in analyzing complex exhaled mixtures.

\section{Challenges of breath analysis}

Breath analysis has been promising and of great interest to researchers in clinical applications. However, the concentration of exhaled endogenous VOCs can vary considerably not only from person to 
person, but also day to day and even breath to breath for the same individual. The cause of most of the breath biomarkers is still not recognized and many are under the subject of intense research. Also, there is a lack of standardized general guidelines or procedures for breath sampling, analysis, and background correction. There are various factors which would affect the breath analysis results:

Route of exhalation: Researchers have demonstrated that concentration levels for some VOCs are affected by the route of exhalation, i.e., mouth, nose and both. It was shown that some VOCs could be produced in the oral cavity instead of endogenously exchanged on the alveolar interface $[49,75]$. Therefore, the route of exhalation may affect the measured concentration of the analytes of interest. Moreover, collection of breath sample can be done in a single breath but more accurate results can be done in averaging a series of breath samples [36].

Dead space air: Dead space air is the part of inhaled air which does not perform gas exchange and it is usually the portion that stays in the conducting airways during the breathing cycle. Breath sampling is a general sample of the exhaled breath and it is not easy to separate the dead space air. The volumes of breath samples from each patient can be different, therefore, the dead space volume can dilute the endogenous VOCs' concentrations by different degrees. End tidal breath sampling (collection at the end of exhalation) for breath analysis is an interesting technique recently proposed. Certain target molecules from the end tidal breath would be less diluted by the dead space air during the sampling. It has the potential to provide an alternative pre-concentration method without chemical manipulation to the sample to avoid contamination and absorption competition. Researchers have shown the possibility of real-time buffered end tidal measurements of breath VOCs followed by MS-based techniques [18].

Personal/medical issues: The difference in concentration levels of some types of exhaled molecules can vary across gender, age, diet, drug and other medical conditions $[14,60,64]$. The fact that some patients are less able to offer full exhalation should be taken into account. For instance, patients with late-stage lung cancer may only be able to offer a small vital capacity due to the weak exhalation force. As inhaled air sitting at more upper airways would be exhaled before the lower airways, the molecules released by a deeper sitting tumour would be less exposed to fresh air and less likely to be exhaled compared to those from the upper airways.

Measuring tools: To measure ultra-low concentrations, the testing tools must be cleaned and sterilized to remove potential contaminants, e.g., residues from the manufacturing process, cleaning agents, etc. To make sure the endogenous VOCs from the alveolar breath are measured and distinguished, some other common compounds that could be found in the oral cavity may also need to be eliminated during the analysis, e.g., oral hygiene products, food. Sample manipulation (such as pre-concentration) may also introduce calculation error due to selective/non-linear absorption.

The combination of the exhaled VOCs and their corresponding concentrations could vary significantly between different sample sources and instrumental responses. The large variation of results in the literature for the same test is one of the major problems preventing breath testing from being widely applied in the medical field. The background can generally be minimized by subtraction and supplying air for inhalation with known concentration of VOCs. However, these processes are less convincing if the changes in concentration of VOCs are tiny compared to the background level. Moreover, complex issues such as dead space volume are hard to correct for via simple subtraction. As a result, data from breath analysis usually show wide variation. More effort has to be made in this area to provide adequate standardized procedures for breath sampling, analysis and background correction, to allow for reliable comparisons between different published results. 


\section{Conclusion}

Breath analysis is a promising disease screening and diagnostic tool as it can offer real-time, noninvasive and cost-effective analysis. Breath analysis for medical applications is in its infancy as there are lots of unexplored areas such as a solid confirmation for the origin of the exhaled disease biomarkers. Although researchers have been seeking pathophysiological explanations for the observed breath biomarker-disease correlations, more research is needed for further developments in this field.

Analytical experimentalists have been trying to perform breath analysis with various spectroscopic techniques: MS-based, LAS-based and other types such as Raman spectroscopy-based breath analyzer. MS-based tools are reliable, sensitive and versatile for molecular identification and quantification. However, they are less affordable and not easily compactable. So it limits the applicability of MS-based tools to the frontline clinical applications. Laser-based technologies are usually faster, simpler and more costeffective. Optical systems of LAS are usually more compact and the construction is less complicated than most MS-based systems. However, LAS-based tools are commonly limited by the spectral range and therefore it is not easy to detect complex exhaled mixtures simultaneously.

A simple, affordable and point-of-care breath analysis technique for clinical use has been the goal in breath analysis. Most of the spectroscopic techniques for breath analysis have achieved on-line, realtime measurements of exhaled substances with the LOD in the pptv range. This promising field has been growing remarkably with improving technologies in sampling, detection and analysis. Breath analysis has the potential to one day help us in clinical patient monitoring, disease screening and diagnosis, providing better quality of medical services to society on a global scale.

\section{Acknowledgement}

This work is funded by the Canadian Cancer Society (grant \#2012-701364).

\section{References}

[1] A. Bajtarevic, C. Ager, M. Pienz, M. Klieber, K. Schwarz, M. Ligor, T. Ligor, W. Filipiak, H. Denz, M. Fiegl, W. Hilbe, W. Weiss, P. Lukas, H. Jamnig, M. Hackl, A. Haidenberger, B. Buszewski, W. Miekisch, J. Schubert and A. Amann, Noninvasive detection of lung cancer by analysis of exhaled breath, BMC Cancer 9 (2009), 348-364.

[2] Y.A. Bakhirkin, A.A. Kosterev, R.F. Curl, F.K. Tittel, D.A. Yarekha, L. Hvozdara, M. Giovannini and J. Faist, Subppbv nitric oxide concentration measurements using cw thermoelectrically cooled quantum cascade laser-based integrated cavity output spectroscopy, Applied Physics B - Lasers and Optics 82 (2006), 149-154.

[3] J.I. Baumbach, Ion mobility spectrometry coupled with multi-capillary columns for metabolic profiling of human breath, Journal of Breath Research 3 (2009), 034001.

[4] R.S. Blake, P.S. Monks and A.M. Ellis, Proton-transfer reaction mass spectrometry, Chemical Reviews 109 (2009), 861-896.

[5] P. Čáp, K. Dryahina, F. Pehal and P. Španěl, Selected ion flow tube mass spectrometry of exhaled breath condensate headspace, Rapid Communications in Mass Spectrometry 22 (2008), 2844-2850.

[6] W.H. Cheng and W.J. Lee, Technology development in breath microanalysis for clinical diagnosis, Journal of Laboratory and Clinical Medicine 133 (1999), 218-228.

[7] H.-J. Cho, K. Baek, H.-H. Lee, S.-H. Lee and J.-W. Yang, Competitive extraction of multi-component contaminants in water by Carboxen-polydimethylsiloxane fiber during solid-phase microextraction, Journal of Chromatography A 988 (2003), 177-184.

[8] A. Critchley, T.S. Elliott, G. Harrison, C.A. Mayhew, J.M. Thompson and T. Worthington, The proton transfer reaction mass spectrometer and its use in medical science: Applications to drug assays and the monitoring of bacteria, International Journal of Mass Spectrometry 239 (2004), 235-241. 
[9] E.R. Crosson, K.N. Ricci, B.A. Richman, F.C. Chilese, T.G. Owano, R.A. Provencal, M.W. Todd, J. Glasser, A.A. Kachanov, B.A. Paldus, T.G. Spence and R.N. Zare, Stable isotope ratios using cavity ring-down spectroscopy: Determination of C-13/C-12 for carbon dioxide in human breath, Anal. Chem. 74 (2002), 2003-2007.

[10] H. Dahnke, D. Kleine, P. Hering and M. Murtz, Real-time monitoring of ethane in human breath using mid-infrared cavity leak-out spectroscopy, Applied Physics B - Lasers and Optics 72 (2001), 971-975.

[11] S. Davies, P. Spanel and D. Smith, Quantitative analysis of ammonia on the breath of patients in end-stage renal failure, Kidney International 52 (1997), 223-228.

[12] S. Davies, P. Spanel and D. Smith, A new 'online' method to measure increased exhaled isoprene in end-stage renal failure, Nephrology Dialysis Transplantation 16 (2001), 836-839.

[13] C.H. Deng, J. Zhang, X.F. Yu, W. Zhang and X.M. Zhang, Determination of acetone in human breath by gas chromatography-mass spectrometry and solid-phase microextraction with on-fiber derivatization, Journal of Chromatography B - Analytical Technologies in the Biomedical and Life Sciences 810 (2004), 269-275.

[14] M.J. Epton, K. Ledingham, J. Dummer, W.P. Hu, B. Rhodes, S.T. Senthilmohan, J.M. Scotter, R. Allardyce, J. Cook and M.P. Swanney, Interference of chlorofluorocarbon (CFC)-containing inhalers with measurements of volatile compounds using selected ion flow tube mass spectrometry, Rapid Communications in Mass Spectrometry 23 (2009), 443-447.

[15] M. Giardina and S.V. Olesik, Application of low-temperature glassy carbon-coated macrofibers for solid-phase microextraction analysis of simulated breath volatiles, Anal. Chem. 75 (2003), 1604-1614.

[16] C. Grote and J. Pawliszyn, Solid-phase microextraction for the analysis of human breath, Anal. Chem. 69 (1997), 587-596.

[17] A. Hansel, A. Jordan, R. Holzinger, P. Prazeller, W. Vogel and W. Lindinger, Proton-transfer reaction mass-spectrometry Online trace gas-analysis at the ppb level, International Journal of Mass Spectrometry 149 (1995), 609-619.

[18] J. Herbig, T. Titzmann, J. Beauchamp, I. Kohl and A. Hansel, Buffered end-tidal (BET) sampling - A novel method for real-time breath-gas analysis, Journal of Breath Research 2 (2008), 037008.

[19] E.P.L. Hunter and S.G. Lias, Evaluated gas phase basicities and proton affinities of molecules: An update, Journal of Physical and Chemical Reference Data 27 (1998), 413-656.

[20] R. Hyspler, S. Crhova, J. Gasparic, Z. Zadak, M. Cizkova and V. Balasova, Determination of isoprene in human expired breath using solid-phase microextraction and gas chromatography-mass spectrometry, J. Chromatogr. B - Biomed. Sci. Appl. 739 (2000), 183-190.

[21] A. Jordan, S. Haidacher, G. Hanel, E. Hartungen, J. Herbig, L. Mark, R. Schottkowsky, H. Seehauser, P. Sulzer and T.D. Mark, An online ultra-high sensitivity Proton-transfer-reaction mass-spectrometer combined with switchable reagent ion capability (PTR+SRI-MS), International Journal of Mass Spectrometry 286 (2009), 32-38.

[22] S.S. Kim, C. Young, J. Chan, C. Carter and B. Mizaikoff, Hollow waveguide gas sensor for mid-infrared trace gas analysis, in: 2007 IEEE Sensors, Vols 1-3, 2007, pp. 1267-1270.

[23] C.M.F. Kneepkens, G. Lepage and C.C. Roy, The potential of the hydrocarbon breath test as a measure of lipidperoxidation, Free Radical Biology and Medicine 17 (1994), 127-160.

[24] A.A. Kosterev, F.K. Tittel, D.V. Serebryakov, A.L. Malinovsky and I.V. Morozov, Applications of quartz tuning forks in spectroscopic gas sensing, Review of Scientific Instruments 76 (2005), 043105.

[25] I. Kushch, B. Arendacka, S. Stolc, P. Mochalski, W. Filipiak, K. Schwarz, L. Schwentner, A. Schmid, A. Dzien, M. Lechleitner, V. Witkovsky, W. Miekisch, J. Schubert, K. Unterkofler and A. Amann, Breath isoprene - Aspects of normal physiology related to age, gender and cholesterol profile as determined in a proton transfer reaction mass spectrometry study, Clinical Chemistry and Laboratory Medicine 46 (2008), 1011-1018.

[26] M. Ligor, T. Ligor, A. Bajtarevic, C. Ager, M. Pienz, M. Klieber, H. Denz, M. Fiegl, W. Hilbe, W. Weiss, P. Lukas, H. Jamnig, M. Hackl, B. Buszewski, W. Miekisch, J. Schubert and A. Amann, Determination of volatile organic compounds in exhaled breath of patients with lung cancer using solid phase microextraction and gas chromatography mass spectrometry, Clinical Chemistry and Laboratory Medicine 47 (2009), 550-560.

[27] W. Lindinger, A. Hansel and A. Jordan, On-line monitoring of volatile organic compounds at pptv levels by means of proton-transfer-reaction mass spectrometry (PTR-MS) - Medical applications, food control and environmental research, International Journal of Mass Spectrometry 173 (1998), 191-241.

[28] W. Lindinger, A. Hansel and A. Jordan, Proton-transfer-reaction mass spectrometry (PTR-MS): On-line monitoring of volatile organic compounds at pptv levels, Chemical Society Reviews 27 (1998), 347-354.

[29] P. Lirk, F. Bodrogi, H. Raifer, K. Greiner, H. Ulmer and J. Rieder, Elective haemodialysis increases exhaled isoprene, Nephrology Dialysis Transplantation 18 (2003), 937-941.

[30] R.F. Machado, D. Laskowski, O. Deffenderfer, T. Burch, S. Zheng, P.J. Mazzone, T. Mekhail, C. Jennings, J.K. Stoller, J. Pyle, J. Duncan, R.A. Dweik and S.C. Erzurum, Detection of lung cancer by sensor array analyses of exhaled breath, American Journal of Respiratory and Critical Care Medicine 171 (2005), 1286-1291.

[31] A. Manolis, The diagnostic potential of breath analysis, Clinical Chemistry 29 (1983), 5-15.

[32] R.M. Matthew, B. Yury, W. Gerard, L. Rafal and K.T. Frank, Recent advances of laser-spectroscopy-based techniques for applications in breath analysis, Journal of Breath Research 1 (2007), 014001. 
[33] M. Mazurenka, A.J. Orr-Ewing, R. Peverall and G.A.D. Ritchie, 4 Cavity ring-down and cavity enhanced spectroscopy using diode lasers, Annual Reports Section "C" (Physical Chemistry) 101 (2005), 100-142.

[34] P.J. Mazzone, Exhaled breath volatile organic compound biomarkers in lung cancer, Journal of Breath Research 6 (2012), 027106.

[35] M. McCulloch, T. Jezierski, M. Broffman, A. Hubbard, K. Turner and T. Janecki, Diagnostic accuracy of canine scent detection in early- and late-stage lung and breast cancers, Integr. Cancer Ther. 5 (2006), 30-39.

[36] W. Miekisch, J.K. Schubert and G.F.E. Noeldge-Schomburg, Diagnostic potential of breath analysis - Focus on volatile organic compounds, Clinica Chimica Acta 347 (2004), 25-39.

[37] B. Moser, F. Bodrogi, G. Eibl, M. Lechner, J. Rieder and P. Lirk, Mass spectrometric profile of exhaled breath - Field study by PTR-MS, Respiratory Physiology and Neurobiology 145 (2005), 295-300.

[38] R. Mukhopadhyay, Don't waste your breath. Researchers are developing breath tests for diagnosing diseases, but how well do they work?, Anal. Chem. 76 (2004), 273A-276A.

[39] K. Namjou, C.B. Roller and G. McMillen, Breath-analysis using mid-infrared tunable laser spectroscopy, in: Sensors, 2007 IEEE, 2007, pp. 1337-1340.

[40] K. Namjou, C.B. Roller, T.E. Reich, J.D. Jeffers, G.L. McMillen, P.J. McCann and M.A. Camp, Determination of exhaled nitric oxide distributions in a diverse sample population using tunable diode laser absorption spectroscopy, Applied Physics B-Lasers and Optics 85 (2006), 427-435.

[41] L.R. Narasimhan, W. Goodman and C.K.N. Patel, Correlation of breath ammonia with blood urea nitrogen and creatinine during hemodialysis, Proceedings of the National Academy of Sciences of the United States of America $\mathbf{9 8}$ (2001), $4617-4621$

[42] Y. Okita, T. Katagiri and Y. Matsuura, A Raman cell based on hollow optical fibers for breath analysis, in: Optical Fibers and Sensors for Medical Diagnostics and Treatment Applications X, I. Gannot, ed., SPIE - Int. Soc. Optical Engineering, Bellingham, 2010.

[43] B.A. Paldus and A.A. Kachanov, An historical overview of cavity-enhanced methods, Canadian Journal of Physics $\mathbf{8 3}$ (2005), 975-999.

[44] L. Pauling, A.B. Robinson, R. Teranish and P. Cary, Quantitative analysis of urine vapor and breath by gas-liquid partition chromatography, Proceedings of the National Academy of Sciences of the United States of America 68 (1971), 2374-2376.

[45] M. Phillips, Breath tests in medicine, Scientific American 267 (1992), 74-79.

[46] M. Phillips, R.N. Cataneo, A.R.C. Cummin, A.J. Gagliardi, K. Gleeson, J. Greenberg, R.A. Maxfield and W.N. Rom, Detection of lung cancer with volatile markers in the breath, Chest 123 (2003), 2115-2123.

[47] M. Phillips, K. Gleeson, J.M.B. Hughes, J. Greenberg, R.N. Cataneo, L. Baker and W.P. McVay, Volatile organic compounds in breath as markers of lung cancer: A cross-sectional study, Lancet 353 (1999), 1930-1933.

[48] M. Phillips, J. Herrera, S. Krishnan, M. Zain, J. Greenberg and R.N. Cataneo, Variation in volatile organic compounds in the breath of normal humans, Journal of Chromatography B: Biomedical Sciences and Applications 729 (1999), 75-88.

[49] A. Pysanenko, P. Spanel and D. Smith, A study of sulfur-containing compounds in mouth- and nose-exhaled breath and in the oral cavity using selected ion flow tube mass spectrometry, Journal of Breath Research 2 (2008), 046004.

[50] J. Rieder, P. Lirk, C. Ebenbichler, G. Gruber, P. Prazeller, W. Lindinger and A. Amann, Analysis of volatile organic compounds: Possible applications in metabolic disorders and cancer screening, Wiener Klinische Wochenschrift 113 (2001), $181-185$

[51] T.H. Risby and S.F. Solga, Current status of clinical breath analysis, Applied Physics B - Lasers and Optics 85 (2006), 421-426.

[52] C. Roller, K. Namjou, J. Jeffers, W. Potter, P.J. McCann and J. Grego, Simultaneous NO and $\mathrm{CO}_{2}$ measurement in human breath with a single IV-VI mid-infrared laser, Opt. Lett. 27 (2002), 107-109.

[53] V. Ruzsanyi, J.I. Baumbach, S. Sielemann, P. Litterst, M. Westhoff and L. Freitag, Detection of human metabolites using multi-capillary columns coupled to ion mobility spectrometers, Journal of Chromatography A 1084 (2005), 145-151.

[54] V. Savarino, S. Vigneri and G. Celle, The C-13 urea breath test in the diagnosis of Helicobacter pylori infection, Gut 45 (1999), I18-I22.

[55] K. Schwarz, W. Filipiak and A. Amann, Determining concentration patterns of volatile compounds in exhaled breath by PTR-MS, Journal of Breath Research 3 (2009), 027002.

[56] S.S. Sehnert, L. Jiang, J.F. Burdick and T.H. Risby, Breath biomarkers for detection of human liver diseases: Preliminary study, Biomarkers 7 (2002), 174-187.

[57] M.W. Sigrist, R. Bartlome, D. Marinov, J.M. Rey, D.E. Vogler and H. Wachter, Trace gas monitoring with infrared laserbased detection schemes, Applied Physics B - Lasers and Optics 90 (2008), 289-300.

[58] K.D. Skeldon, L.C. McMillan, C.A. Wyse, S.D. Monk, G. Gibson, C. Patterson, T. France, C. Longbottom and M.J. Padgett, Application of laser spectroscopy for measurement of exhaled ethane in patients with lung cancer, Respiratory Medicine 100 (2006), 300-306.

[59] D. Smith and P. Spanel, Selected ion flow tube mass spectrometry (SIFT-MS) for on-line trace gas analysis, Mass Spectrometry Reviews 24 (2005), 661-700. 
[60] D. Smith, P. Spanel and S. Davies, Trace gases in breath of healthy volunteers when fasting and after a protein-calorie meal: A preliminary study, Journal of Applied Physiology 87 (1999), 1584-1588.

[61] D. Smith, P. Spanel, B. Enderby, W. Lenney, C. Turner and S.J. Davies, Isoprene levels in the exhaled breath of 200 healthy pupils within the age range 7-18 years studied using SIFT-MS, Journal of Breath Research 4 (2010), 017101.

[62] D. Smith, C. Turner and P. Spanel, Volatile metabolites in the exhaled breath of healthy volunteers: Their levels and distributions, Journal of Breath Research 1 (2007), 014004.

[63] G. Song, T. Qin, H. Liu, G.-B. Xu, Y.-Y. Pan, F.-X. Xiong, K.-S. Gu, G.-P. Sun and Z.-D. Chen, Quantitative breath analysis of volatile organic compounds of lung cancer patients, Lung Cancer 67 (2010), 227-231.

[64] P. Spanel, K. Dryahina and D. Smith, The concentration distributions of some metabolites in the exhaled breath of young adults, Journal of Breath Research 1 (2007), 026001.

[65] P. Spanel, Y.F. Ji and D. Smith, SIFT studies of the reactions of $\mathrm{H}_{3} \mathrm{O}^{+}, \mathrm{NO}^{+}$and $\mathrm{O}_{2}^{+}$with a series of aldehydes and ketones, International Journal of Mass Spectrometry 165 (1997), 25-37.

[66] P. Spanel and D. Smith, SIFT studies of the reactions of $\mathrm{H}_{3} \mathrm{O}^{+}, \mathrm{NO}^{+}$and $\mathrm{O}_{2}^{+}$with a series of alcohols, International Journal of Mass Spectrometry 167 (1997), 375-388.

[67] P. Spanel and D. Smith, Selected ion flow tube studies of the reactions of $\mathrm{H}_{3} \mathrm{O}^{+}, \mathrm{NO}^{+}$and $\mathrm{O}_{2}^{+}$with several aromatic and aliphatic hydrocarbons, International Journal of Mass Spectrometry 181 (1998), 1-10.

[68] P. Spanel and D. Smith, Selected ion flow tube mass spectrometry for on-line trace gas analysis in biology and medicine, European Journal of Mass Spectrometry 13 (2007), 77-82.

[69] P. Spanel and D. Smith, Progress in SIFT-MS: Breath analysis and other applications, Mass Spectrometry Reviews 30 (2011), 236-267.

[70] M.J. Thorpe, D. Balslev-Clausen, M.S. Kirchner and J. Ye, Cavity-enhanced optical frequency combspectroscopy: Application to human breathanalysis, Opt. Express 16 (2008), 2387-2397.

[71] C. Wang and P. Sahay, Breath analysis using laser spectroscopic techniques: Breath biomarkers, spectral fingerprints, and detection limits, Sensors 9 (2009), 8230-8262.

[72] C. Wang and A.B. Surampudi, An acetone breath analyzer using cavity ringdown spectroscopy: An initial test with human subjects under various situations, Measurement Science and Technology 19 (2008), 105604.

[73] C.J. Wang, A. Mbi and M. Shepherd, A study on breath acetone in diabetic patients using a cavity ringdown breath analyzer: Exploring correlations of breath acetone with blood glucose and glycohemoglobin A1C, IEEE Sensors Journal 10 (2010), 54-63.

[74] C.J. Wang, S.T. Scherrer and D. Hossain, Measurements of cavity ringdown spectroscopy of acetone in the ultraviolet and near-infrared spectral regions: Potential for development of a breath analyzer, Applied Spectroscopy 58 (2004), 784-791.

[75] T.S. Wang, A. Pysanenko, K. Dryahina, P. Spanel and D. Smith, Analysis of breath, exhaled via the mouth and nose, and the air in the oral cavity, Journal of Breath Research 2 (2008), 037013.

[76] A. Wehinger, A. Schmid, S. Mechtcheriakov, M. Ledochowski, C. Grabmer, G.A. Gastl and A. Amann, Lung cancer detection by proton transfer reaction mass-spectrometric analysis of human breath gas, International Journal of Mass Spectrometry 265 (2007), 49-59.

[77] H. Yu, L. Xu and P. Wang, Solid phase microextraction for analysis of alkanes and aromatic hydrocarbons in human breath, J. Chromatogr. B Analyt. Technol. Biomed. Life Sci. 826 (2005), 69-74. 